\title{
HISTÓRIA DA OBESIDADE NA CLASSIFICAÇÃO INTERNACIONAL DE DOENÇAS (CID): DE 1900 A 2018
}

\author{
Cezar Barbosa Santolin ${ }^{1}$
}

SANTOLIN, C. B. História da obesidade na classificação internacional de doenças (cid): de 1900 a 2018 . Arquivos de Ciências da Saúde da UNIPAR, Umuarama, v. 25, n. 3, p. 167-172, set./dez. 2021.

RESUMO: O presente artigo teve como objetivo descrever o histórico da inserção da condição clínica denominada atualmente de "obesidade" nas onze revisões da Classificação Internacional de Doenças (CID), publicadas pela Organização Mundial da Saúde (OMS), entre os anos 1900 e 2018. Para tanto, buscou-se pela palavra-chave "obesity" nos documentos, realizando, posteriormente, uma descrição e uma análise da presença, modo de inserção e as mudanças ocorridas ao longo do tempo. Os resultados demonstraram que a condição já foi e continua sendo inserida como sintoma, morbidade, coREVSmorbidade, causa de mortalidade e/ou doença. Concluiu-se que há uma grande inconsistência lógica nos princípios que regem a classificação.

PALAVRAS-CHAVE: Obesidade. História. Classificação.

\section{HISTORY OF OBESITY IN THE INTERNATIONAL CLASSIFICATION OF DISEASES (ICD): 1900 TO 2018}

\begin{abstract}
This article aimed at describing the history of insertion of the clinical condition currently referred to as "obesity" in the eleven revisions of the International Classification of Diseases (ICD) published by the World Health Organization (WHO) between 1900 and 2018. For this purpose, a search for the keyword obesity was performed in the documents, with subsequent description and analysis of the presence, mode of insertion, and changes occurring over time. The results demonstrated that the condition has been and continues to be inserted as symptom, morbidity, comorbidity, cause of mortality and/or disease. It can be concluded that there is a massive logical inconsistency in the principles that govern the classification.
\end{abstract}

KEY WORDS: Obesity. History. Classification.

Introdução

A obesidade é conceituada, atualmente, como um acúmulo excessivo de gordura corporal, definido por meio de parâmetros do Índice de Massa Corporal (IMS). A condição é considerada uma doença na área da saúde, que é capitaneada pela Organização Mundial da Saúde (OMS). Esse status patológico acarreta uma série de consequências, que vão desde a legislação até o modo como as pessoas são tratadas. Também por conta disso, a temática tornou-se alvo de infindáveis pesquisas e políticas públicas de saúde - tanto no Brasil quanto no restante do mundo. Mas, como ressaltado por Seixas e Birman (2012, p.14), "apesar da vasta produção médico-científica a respeito da obesidade, em poucas obras podemos identificar claramente sua construção histórica como uma categoria clínica".

Parte da história não somente das primeiras tentativas de sistematização nosológica, proveniente de Sauvages e Cullen² (WHO, 2018a), no Século XVIII, mas também do nascimento de uma problematização patológica do que, posteriormente, veio a ser denominado obesidade, foi descrita em Santolin (2012). Ainda há, entretanto, muitas lacunas a serem preenchidas na história da patologização da obesidade.

Ainda que algumas histórias da obesidade, como aquelas contadas por Repetto (1998), Haslam (2007) e Bray (2009), projetem anacronicamente a patologização da condição em tempos mui remotos - até mesmo na pré-história - pesquisas mais rigorosas, como Vigarello (2012) e outros, tem evidenciado que o processo histórico de patologização foi bem mais recente, situando-se por volta dos Séculos XVIII e XIX.

Nesse sentido, a análise do histórico do que veio a se tornar a Classificação Internacional das Doenças (CID), que guiaria e continua a guiar a Organização Mundial da Saúde (OMS), permite rastrear temporalmente o momento em que a obesidade recebeu o status de doença por essa autoridade médica internacional, cujas publicações são admitidas como oficiais no sistema de saúde de muitos países, tal como o Sistema Único de Saúde (SUS), no Brasil.

A história das CIDs se mistura com a história da nosologia - a taxonomia das doenças. As primeiras tentativas de sistematização formal em listas das condições existentes começaram no Século XIX. William Farr (18071883) rejeitou a nosologia de Cullen, vigente na época, para compor uma estatística médica das doenças com informações provenientes da Bills of Mortality, na Inglaterra (WHO, 2018a). Ainda que as listas produzidas nunca tenham sido universalmente aceitas, "o arranjo proposto por Farr, incluindo o princípio de classificar doenças por região anatômica, sobreviveu como base da Lista Internacional de Causas da Morte" (LICM) (WHO, 2018a).

A LICM será, posteriormente, transformada em CID. A primeira LICM foi elaborada por Jacques Bertillon (1851-1922), ficando conhecida, inclusive, como a "A

DOI: https://doi.org/10.25110/arqsaude.v25i3.2021.8045

${ }^{1}$ Universidade Federal de Mato Grosso do Sul (UFMS). Bacharel, mestre e doutorando em Educação Física. cezarsantolin@hotmail.com. https://orcid. org/0000-0002-4031-6486

${ }^{2}$ François Bossier de Lacroix (1707-1777), estatística australiano, mais conhecido como Sauvages, realizou a primeira tentativa de sistematização das doenças em Nosologia Methodica. Já William Cullen (1710-1790), médico escocês, publicou em 1785 Synopsis nosologiae methodicae. 
classificação de Bertillon das causas da morte". Bertillon ficou à frente das comissões de elaboração das listas nas três primeiras edições (WHO, 2018a)

Assim - um detalhe importante a destacar - as listas que originaram as CIDs não vieram de sistemas nosológicos, mas, sim, dos registros de causas de mortalidade (WHO, 2018a). Esse aspecto histórico marcou a CID, criando uma confusão entre causas de morte e doenças, o que já foi alvo de muitas críticas, como se pode notar na passagem abaixo, da própria OMS:

Enquanto três séculos contribuíram com alguma coisa para a precisão científica da classificação de doenças, há muitos que duvidam da utilidade das tentativas de compilar estatísticas de doenças, ou mesmo causas de morte, devido às dificuldades de classificação. (WHO, 2018a)

Assim, a quantificação estatística epidemiológica depende da classificação, e esta depende de um sistema universalmente aceito de codificação e princípios lógicos consistentes na classificação - nem sempre aceitos unanimemente pela comunidade científica e pela área da saúde.

O problema classificatório ganha contornos mais críticos devido à vinculação aos sistemas legais, através dos sistemas de saúde, como o SUS. No Brasil, desde a criação do SUS, em 1988, adotou-se as CIDs como classificação oficial. Para várias situações médico-legais, exige-se o "número da CID", tornando tal lista uma ferramenta poderosa de biopoder (FOUCAULT, 2008).

Um auxílio doença ou uma aposentadoria por invalidez junto ao Instituto Nacional do Seguro Social (INSS) só são concedidos aos contribuintes se as condições patológicas forem caracterizadas através da CID. Apesar disso, há incoerências quando a questão é a obesidade, pois apesar de ser atualmente classificada como doença na CID vigente, não se tem notícia de contribuintes beneficiados com auxílio doença ou aposentadoria por invalidez devido à obesidade.

Isso não impediu, entretanto, que em outras situações, como em exames médicos para concursos públicos, candidatos tenham sido excluídos por terem sido caracterizados como doentes, utilizando como recurso a presença da condição na CID (FOLHA DE SÃO PAULO, 2018).

Por isso, em vista da importância da articulação entre medicina e direito se dar à luz de tais listas, com potencial para atingir, na prática, a todos, além de poder elucidar o processo histórico de patologização da obesidade, no mundo e no Brasil, o presente artigo teve por objetivo descrever a inserção dessa condição nessas listas de classificação de doenças, desde a criação até a CID atual.

\section{Métodos}

As características da presente pesquisa caracterizamna como histórica e descritiva (STRUNA, 2007; THOMAS;
NELSON; SILVERMAN, 2007). Os documentos históricos que compuseram o corpus empiricus foram as onze edições da CID, cujo acesso foi obtido através do site Wolfbane Cybernetic (2018) e do site ICD-11 (WHO, 2018).

Todas as edições acessadas estavam no idioma original, inglês. As condições de interesse para a presente pesquisa foram traduzidas livremente para o português pelo autor, por meio de consultas a dicionários, quando necessário, buscando resguardar a literalidade e a especificidade dos termos médicos empregados.

Após o acesso aos materiais, realizou-se a consulta em busca de termos médicos já usados ou ainda em uso para se referir à obesidade, tais como: obesity, corpulency, fat, dentre outros. Enquanto as listas ainda eram "pequenas", ou seja, com centenas de condições (até a LICM-5), esta consulta foi realizada item a item. Após atingirem milhares (da CID-6 em diante), buscaram-se tais termos através das ferramentas de busca de softwares de edição de textos, como o Word, e leitor de $p d f$, como o Foxit reader.

Os resultados encontrados foram descritos, com seus respectivos códigos, buscando explicar de que modo a condição se inseria na hierarquia listada, além de descrever possíveis mudanças no modo de inserção da condição ao longo das onze revisões. Posteriormente as descrições, empreenderam-se análises e discussão dos resultados encontrados, conforme o que já se conhece da história da obesidade.

\section{Resultados e discussão}

A história dessas publicações começa em 1900, com a primeira edição de uma Lista Internacional de Causas de Morte (LICM) - nome que se manteria até 1948 - trazendo, ao total, 191 condições listadas. Não há uma estratificação ou hierarquização taxonômica - as condições são listadas e codificadas numericamente, de modo simples.

Ao consultar essa pequena lista, nenhuma das condições se refere à obesidade, excesso de corpulência, Índice de Massa Corporal (IMC), peso ou gordura corporal. A omissão, nesse caso, permite cogitar algumas hipóteses igualmente plausíveis. Pode-se deduzir através desse documento, portanto, que em 1900: a) ou tais condições não seriam consideradas realmente causas de morte; b) ou, se fossem, seriam identificadas com algum rótulo geral/inespecífico; c) ou não havia número significativo epidemiologicamente de ocorrências (prevalência insignificante).

Consta na lista, entretanto, a fome (56) como, sugerindo que a escassez de comida seria um problema mais preocupante, ocasionando, inclusive, óbitos, do que possíveis consequências de excessos alimentares.

Itens, aparentemente, curiosos presente na LICM-1, como velhice $^{3}$ (167) e uma série de mortes violentas (175191), podem ser entendidos se levarmos em consideração que a lista seria uma relação de "causas de morte", ou seja, direcionada mais para médicos legistas ou seguros de vida do que para clínicos e especialistas.

\footnotetext{
${ }^{3}$ Do inglês old age.
} 
Apesar disso, destaca-se, ainda, nesta edição a presença de condições que, paradoxalmente, não levam à morte, como convulsões (89) e epilepsia (88). Aparentemente, essa prévia de confusão em torno da diferença entre causa, sintoma, forma de contágio, prognóstico, traumas, patologias, entre outros, manter-se-á e aumentará exponencialmente, ao longo das revisões, o número de condições listadas.

Em 1909 foi publicada a obra revisada, onde o número de condições ascende para 319 - um aumento de quase $70 \%$, mas uma quantidade diminuta se compararmos ao tamanho que tais listas ficarão próximo ao final do Século XX. Apesar disso, nessa já há uma estratificação simples das condições, que são codificadas com um número e, quando há subcategorias, com letras maiúsculas. Em alguns casos, existem até oito subcategorias.

Novamente, ao consultar a lista, nenhuma das condições se referia aos itens que poderiam se referir a um excesso de gordura corporal, peso, IMC, tamanho do corpo, etc. A fome (177), entretanto, permanece na lista. E, mais uma vez, também, várias condições que não levariam ao óbito, como fimose (150-B), podem ser encontradas.

A terceira publicação, de 1920, traz uma pequena redução da lista para 315 . O esquema de estruturação tornase mais complexo - letras minúsculas definem subgrupos e números definem subcategorias.

Na LICM-3 é possível encontrar a condição denominada "coração gordo"4 [90 (9)], que seria a primeira aparição da gordura excessiva como agente etiológico de uma condição que poderia acarretar em óbito. Excetuando esse caso, assim como nas demais listas anteriores, nada consta dentre os itens que remeta ao conceito de excesso de gordura corporal ou obesidade. Dessa vez, aparece o item "fome ou sede" (192).

Em 1929 será lançada a quarta edição revisada da LICM, com o total de 331 condições listadas. Permanece a condição denominada "coração gordo" [93b (1)] e, também, a "fome ou sede" (189). O esquema de estratificação da lista segue o padrão da revisão anterior.

A quinta revisão, de 1938, conteve 487 condições classificadas. Nada constou, entretanto, sobre condições relacionadas ao excesso de gordura corporal. Mesmo a condição denominada "coração gordo", presente nas duas revisões anteriores, deixa de constar na lista.

Em 1948, ano em que a OMS foi fundada, ocorre a mudança do nome de Lista Internacional de Causas de Morte para Classificação Internacional de Doenças (CID). Originalmente, fora intitulada "International Classification of Diseases, Injuries, and Causes of Death" (Classificação Internacional de Doenças, Lesões e causas de morte) (WHO, 2018a). Estruturalmente, mantém-se uma lista estratificada, mas com quatro dígitos numéricos e - em alguns subgrupos - com uma letra inicial maiúscula.

Apesar de a publicação ser, de fato, uma continuação da outra - tanto é que, dando sequência à série, nascerá como CID-6 - a pequena mudança do título de "causa de mortes" para "doenças" tem uma implicação significativa.

O primeiro aspecto a ressaltar é que nem toda causa

${ }^{4}$ Fatty heart. de morte pode advir de algo considerado uma doença - e vice-versa. Uma grande parte das condições trazidas pela CID-6 é, na verdade, uma causa de morte e não poderia ser, de forma alguma, patologizada. Por exemplo, uma ampla gama de acidentes, presentes nas LICMs, continua na lista, sugerindo que tais traumas seriam doenças.

Mas não foi só essa a ruptura em relação às listas anteriores. A OMS adota como conceito de saúde o "estado do mais completo bem-estar físico, mental e social e não apenas a ausência de doença" (SCLIAR, 2007, p.37). Desde então, muitas críticas teriam sido feitas a essa definição, principalmente a alegação de que seria um ideal inatingível e que daria poder ao Estado de intervir na vida dos cidadãos, o que foi caracterizado, contemporaneamente, como um biopoder totalitário (SCLIAR, 2007; FOUCAULT, 2008).

Como Caponi (apud GOMES, 2009) ressalta, a inclusão da noção de bem-estar, sobretudo social, no conceito de saúde se inscreveria no espaço da normalidade, que como Canguilhem (1995) percebeu, confunde-se com a frequência e com a média/moda estatística. Tal abordagem do conceito de saúde encerraria questões valorativas, que deveriam estar sob o poder do indivíduo.

Como a definição do que seria saúde implica, consequentemente, na definição do que seria doença, após a mudança, o número de condições mais que dobra em relação à LICM-5; passando de 487 para mais de 4.500 $(\approx 824 \%$ de aumento). Ou seja, se adotarmos a definição proposta pela OMS, como passa a ser feito na CID-6, há poucas chances de que alguém seja considerado realmente saudável. A "área da saúde", assim, seria melhor denominada como "área da doença". Consequentemente, também, é na CID-6 que aparece, pela primeira vez, tanto na classificação simplificada quanto na expandida, uma patologização oficial da obesidade na história. A condição "obesidade não especificada, de origem endócrina" consta no item 287 e não figura dentro de nenhum subgrupo específico. Não é possível deduzir se a condição foi posta na lista enquanto uma doença ou, simplesmente, enquanto um sintoma de uma disfunção, doença ou patologia endócrina. Há, entretanto, uma espécie de asserção etiológica no item, que sugere que disfunções endócrinas podem causar obesidade.

Dentro da nova definição de saúde proposta pela OMS, o possível raciocínio dos formuladores da lista, aparentemente, foi que - mesmo se a condição não se enquadrasse como um "mal-estar", especificamente, físico ou mental - poderia ser entendido como um bem-estar social incompleto, já que os indivíduos sofrem estigmatização por serem anômalos antropometricamente, e, portanto, deveria ser classificado como doença.

Ressalta-se que em 1948, ano de divulgação da CID-6, não havia evidências clínicas claras de que o excesso de IMC, peso ou gordura corporal fossem variáveis independentes e, principalmente, causais para a associação dessas com as alegações que eram feitas baseadas, exclusivamente, em estudos epidemiológicos longitudinais ou em ensaios clínicos controlados e randomizados. Também não havia uma definição oficial do parâmetro técnico que classificaria alguém como obeso, que só veio a ser definido pela OMS em 1998 (OLIVER, 2006). Aparentemente, a 
"obesidade" referida na lista seria o equivalente da corpulência excessiva - e não com o significado médico atual. Por se tratar somente de uma lista, sem definições conceituais, não é possível somente com esse material elucidar qual seria o real significado da palavra empregada.

A CID-7, lançado em 1955, aumenta um pouco o montante de condições patológicas, totalizando cerca de 4.900 itens listados. A classificação do item que referencia a obesidade se mantém idêntico, inclusive no número de referência, assim como a estrutura geral da lista e o esquema de codificação.

Igualmente, a CID-8 manterá um pequeno acréscimo na lista, que somará aproximadamente, 4.500 condições. A única diferença na classificação da "obesidade não especificada como de origem endócrina" é que o número da classificação passa a ser 277. Em termos estruturais e de codificação, a CID-8 se mantém semelhante às CIDs 6 e 7.

Já na CID-9, publicada em 1975, ocorrem mudanças significativas. A codificação das condições passa a ser realizada com 5 números e se mantém a utilização de letras maiúsculas, em alguns casos, para definir subgrupos de doenças. A lista atinge a marca impressionante de mais de 17.000 itens ( $\approx 278 \%$ de aumento).

A relação entre o aumento do número de condições classificadas como patológicas e o aumento do "público alvo" ou consumidor dos serviços médicos e dos produtos farmacêuticos, parece óbvia o bastante para dispensar maiores aprofundamentos argumentativos. Oliver (2006) se refere a um complexo industrial da saúde, que incluiria, inclusive, a área da Educação Física.

A condição denominada "obesidade e outras hiperalimentações" (278) é encontrado na lista reduzida, que contabiliza 1.188 condições, enquanto na expandida são discriminados os subitens "obesidade" (278.0) e “adiposidade localizada" (278.1). Vale ressaltar que há outras hiperalimentações no subgrupo 278, como hipervitaminoses, o que permite deduzir que o termo "obesidade" empregado era definido mais por uma suposta etiologia (hiperalimentação) do que pela definição conceitual da condição em si.

A condição denominada unicamente de "obesidade" também aparece em V77.8, um subgrupo de "Triagem especial para distúrbios endócrinos, nutricionais, metabólicos e imunológicos" (V77). Dado o exposto no parágrafo anterior, cogita-se que tenha sido acrescida enquanto distúrbio nutricional, de hiperalimentação.

Novamente, nessa época, a OMS não estabelecia parâmetros técnicos de definição do que seria considerado adiposidade localizada ou obesidade. Portanto, caberia ao médico definir se a concentração de tecido adiposo em determinado indivíduo se configuraria ou não em tal quadro.
A OMS também não explica porque tais condições estariam na lista - se seriam doenças, sintomas ou causas de morbimortalidade.

A penúltima publicação revisada, o CID-10 publicada em 1990 e ainda em uso tanto no Brasil como internacionalmente - traz cerca de 14.000 condições consideradas patológicas. A codificação das condições é realizada com 3 caracteres, numa lista simplificada e menor, ou com 4 caracteres, na lista maior. O código é composto por uma letra maiúscula, seguida de dois números, que especificam a condição, seguido por um ponto, e, depois, mais um número, que especifica um subitem.

As condições relacionadas à obesidade encontradas estão dentro de uma categoria denominada “Obesidade e outras hiperalimentações" (E65-E68). As condições diretamente ligadas ao tema deste estudo foram: "adiposidade localizada" (E65), "obesidade" (E66) e "outras hiperalimentações" (E67). Somente a categoria E66 possuía subitens: "obesidade por excesso de calorias" (E66.0), "obesidade induzida por drogas" (E66.1), "obesidade extrema com hipoventilação pulmonar" (E66.2), "outra obesidade” (E66.8) e "obesidade não especificada” (E66.9).

Desde a primeira edição das CIDs, é possível perceber uma grande inconsistência categórica na lógica nosológica. Em alguns itens, nomeia-se uma condição considerada uma doença, enquanto em outros consta aspectos relacionados à etiologia (causa), ao modo de distribuição, sintomas associados, dentre outros aspectos. Cogita-se que se tornaria inviável e inoportuno especificar numa lista de doenças quais as causas, sintomas dentre outros aspectos, de cada doença. Entende-se que uma doença é um constructo conceitual abstrato, produzido por indução, a partir de recorrências de casos específicos, em que se identificou sintomas, causas, consequências, dentre outros aspectos, de uma condição específica, causadora de um certo pathos. Se uma lista que se pretende ser uma lista de todas as doenças possíveis de afetar a humanidade adentrar em questões secundárias, como causa, sintomas, etc. tal empreendimento se tornaria inviável. Haveria, sem dúvida, locais mais oportunos - como os compêndios médicos - para destrinchar as minúcias que uma determinada doença pode ter.

A CID 11 foi publicada em Junho de 2018 e entrará em vigor no SUS a partir de $1^{\circ}$ de Janeiro de 2022. Por estar disponível somente num site específico, que não permite uma consulta da lista completa na íntegra, fica difícil determinar quantas condições constam nesta revisão. A codificação foi realizada com até 6 caracteres, que incluem letras maiúsculas e números.

O termo "obesidade" aparece em 26 condições listadas, conforme é possível consultar no quadro abaixo (Quadro 1):

Quadro 1: Lista de condições e seus respectivos códigos com o termo obesidade na CID-11 (2018)

\begin{tabular}{|c|l|c|}
\hline $\mathbf{n}$ & \multicolumn{1}{|c|}{ Condição listada } & Código \\
\hline 1 & Obesidade, inespecífica & 5B81.Z \\
\hline 2 & Outra hipofunção específica ou desordem da glândula pituitária obesidade & $5 \mathrm{~A} 61 . \mathrm{Y}$ \\
\hline 3 & Obesidade devido a desequilíbrio energético & $5 \mathrm{~B} 81.0$ \\
\hline 4 & Obesidade em crianças ou adolescentes & $5 \mathrm{~B} 81.00$ \\
\hline
\end{tabular}




\begin{tabular}{|c|c|c|}
\hline 5 & Obesidade em adultos & $5 B 81.01$ \\
\hline 6 & Obesidade induzida por droga & 5B81.1 \\
\hline 7 & Outra obesidade específica & 5B81.Y \\
\hline 8 & Linfodema devido à obesidade & BD93.12 \\
\hline 9 & Outra osteoporose específica $>$ osteoporose de obesidade & FB83.1Y \\
\hline 10 & Hipotireoidismo adquirido, não especificado $>$ obesidade hipotireóidea & $5 \mathrm{~A} 00.2 \mathrm{Z}$ \\
\hline 11 & Sobrepeso em adultos $>$ pré obesidade & $5 \mathrm{~B} 80.01$ \\
\hline 12 & Estrias de outra etiologia especificada > Estiramentos relacionados à obesidade & EE40.1Y \\
\hline 13 & Síndrome de hipoventilação por obesidade & 7A42.0 \\
\hline 14 & Desordens neurológicas devido a sobrepeso ou obesidade em adultos ou crianças & $8 \mathrm{D} 42$ \\
\hline 15 & Ganho de peso excessivo na gravidez $>$ síndrome da obesidade maternal & JA65.2 \\
\hline 16 & Síndromes com obesidade como característica principal & LD29 \\
\hline 17 & Outra surdez genética sindrômica especificada $>$ choroideremia - surdez - obesidade & LD2H.Y \\
\hline 18 & $\begin{array}{l}\text { Contato com os serviços de saúde para aconselhamento ou vigilância dietética > aconselhamento } \\
\text { dietético ou vigilância para obesidade }\end{array}$ & QA10 \\
\hline 19 & $\begin{array}{l}\text { Outro aumento da pressão intracraniana especificada > Hipertensão intracraniana associada à } \\
\text { obesidade }\end{array}$ & 8D60.Y \\
\hline 20 & Deficiência de Coenzima Q10 > Surdez - encefalopatia - obesidade - valvopatia & $5 \mathrm{C} 53.22$ \\
\hline 21 & Outro especificado excesso de peso, obesidade ou excesso de nutrientes específicos & $5 \mathrm{C} 1 \mathrm{Z}$ \\
\hline 22 & $\begin{array}{l}\text { Síndromes de supercrescimento > macrossomia - obesidade - macrocefalia - anormalidades } \\
\text { oculares }\end{array}$ & $\mathrm{LD} 2 \mathrm{C}$ \\
\hline 23 & Outro sobrepeso, obesidade ou excesso de nutriente específicos & $5 \mathrm{C} 1 \mathrm{Y}$ \\
\hline 24 & Feto ou recém-nascido afetado por excesso de peso ou obesidade materna & KA00.61 \\
\hline 25 & Acantose benigna nigricans $>$ acanthosis nigricans associada a diabetes tipo II e obesidade & ED51.00 \\
\hline 26 & $\begin{array}{l}\text { Distrofias hereditária da retina }>\text { retardo mental - obesidade troncular - distrofia retiniana - } \\
\text { micropênis }\end{array}$ & $9 \mathrm{~B} 70$ \\
\hline
\end{tabular}

Além do aumento considerável no número de ocorrências de condições com a palavra-chave pesquisada, em comparação às demais CIDs, uma novidade é a presença da condição denominada "pré-obesidade", utilizada como sinônimo de "sobrepeso" na lista. A patologização avança sobre as diversas condições possíveis de vida, limitando o espectro de normalidade, no qual ainda se poderia ser classificado saudável. Assim, oportunamente para alguns, a vida - em sua variabilidade intrínseca - vai se tornando passível de tratamento, tornando todos pacientes em potencial.

Novamente, chama à atenção a incoerência nosológica - às vezes, a obesidade é uma condição patológica, às vezes um sintoma; às vezes, incluem-se aspectos epidemiológicos (subgrupo atingido), às vezes, etiológicos. A diversidade de critérios numa lista classificatória gera a possibilidade de que um mesmo caso seja classificado em dois ou mais itens, dada a lógica $f u z z y^{5}$, causando confusão e falência do objetivo da iniciativa classificatória, que consiste em organizar.

Para exemplificar esse ponto de vista, basta perceber que um mesmo sujeito pode ser classificado - em relação à obesidade - em duas CIDs, tais como "obesidade em adultos" (5B81.01) e "obesidade induzida por droga" (5B81.1), já que o primeiro item listado especifica qual subgrupo epidemiológico e o segundo especifica qual a causa, não havendo, portanto, uma incoerência lógica

${ }^{5}$ Diferentemente da lógica dita nítida ou booleana, em que só se aceita dois valores - verdadeiro ou falso, na lógica difusa ou fuzzy aceita-se valores intermediários múltiplos entre o verdadeiro e o falso. essencial na dupla classificação. Entende-se que classificar é uma atividade complexa, cujo objetivo é a organização, e que um dos princípios classificatórios fundamentais consiste em impedir casos como esses. A lista ideal deve possuir categorias mutuamente excludentes para evitar o confundimento classificatório e permitir a organização.

\section{Conclusões}

O objetivo do presente artigo foi descrever as inserções da condição atualmente denominada obesidade nas listas de classificação de doenças (LICMs e CIDs) ao longo da história. A análise dos dados permitiu concluir que a primeira inserção da condição se deu em 1948, ano de criação da OMS e de mudança do nome da lista de LICM para CID.

Pode-se perceber, também, um aumento considerável no número de condições relacionadas à obesidade desde essa primeira inserção até a última versão, de 2018. Além desse aspecto quantitativo, percebeu-se grande inconsistência lógica nos princípios classificatórios das condições listadas - qualitativamente, pode-se dizer que a lista tem fraquezas lógicas consideráveis.

As ambivalências criadas devido a isso podem servir de pretexto tanto para classificar a obesidade como doença - quando conveniente - como para classificá-la como mero sintoma, restringindo direitos que, porventura, beneficiários poderiam ter junto à sistemas e planos de saúde.

Por isso, reitera-se a relevância do presente estudo, 
do ponto de vista social, ao informar os cidadãos a respeito dessa temática. Do mesmo modo, para pesquisadores da história da obesidade, é fundamental evidenciar, com fontes, que a institucionalização oficial da obesidade enquanto categoria clínica se deu em meados do Século XX, como se pode ver.

Por fim, espera-se que outros estudos semelhantes possam contribuir para preencher as lacunas existentes na história da patologização da obesidade.

\section{Referências}

BRAY, G. A. History of obesity. In: WILLIAMS, G.; FRÜHBECK, G. Obesity: science to practice. Chicester: Wiley-Blackwell, 2009.

CANGUILHEM, G. O normal e o patológico. Rio de Janeiro: Forense, 1995.

FOUCAULT, M. Nascimento da biopolítica: curso dado no Collège de France (1978-1979). São Paulo: Martins Fontes, 2008.

GOMES, I. M. E se Ivan Ilitch fizesse atividade física? Reflexões sobre tormentos modernos. Pensar a Prática, v. 12, n. 1, 2009.

REAVALIADOS, professores são liberados em concurso. Disponível em: http://www1.folha.uol.com.br/ cotidiano/885179-reavaliados-professores-obesos-saoliberados-em-concurso.shtml. Acesso em: 20 ago. 2018.

HASLAM, D. Obesity: a medical history. Obesity reviews. v. 8. Supl. 1, p. 31-36, 2007.

OLIVER, J. E. Fat politics: the real history behind America's obesity epidemic. Nova York: Oxford University, 2006.

REPETTO, G. Histórico da obesidade. In: HALPERN, A. et al. Obesidade. São Paulo: Lemos, 1998.

SANTOLIN, Cezar Barbosa. O nascimento da obesidade: um estudo genealógico do discurso patologizante. Dissertação (Mestrado) - Universidade Federal de Pelotas (UFPel), Pelotas, 2012.

SEIXAS, C. M.; BIRMAN, J. O peso do patológico: biopolítica e a vida nua. História, Ciências, Saúde Manguinhos. Rio de Janeiro, v. 19, n. 1, jan./mar. 2012, p. 13-26.

SCLIAR, M. História do conceito de saúde. PHYSIS: Rev. Saúde Coletiva, Rio de Janeiro, v. 17, n.1, p. 29-41, 2007.

STRUNA, N. L. Pesquisa histórica em atividade física. In: THOMAS, J. R.; NELSON, J. K.; SILVERMAN, S. $\mathrm{J}$. Métodos de pesquisa em atividade física. 5 . ed. Porto
Alegre: Artmed, 2007, p. 189-201.

THOMAS, J. R.; NELSON, J. K.; SILVERMAN, S. J. Métodos de pesquisa em atividade física. 5. ed. Porto Alegre: Artmed, 2007.

VIGARELLO, G. As metamorfoses do gordo. Rio de Janeiro: Vozes, 2012.

WOLFBANE cybernetic. Disponível em: http://www. wolfbane.com/icd/. Acesso em: 03 jul. 2018.

WORLD HEALTH ORGANIZATION (WHO).

International Classification of diseases: revision 2. 1909. Disponível em: http://www.wolfbane.com/icd/icd2h.htm. Acesso em: 03 jun. 2018.

WORLD HEALTH ORGANIZATION (WHO).

International Classification of diseases: revision 3. 1920. Disponível em: http://www.wolfbane.com/icd/icd3h.htm. Acesso em: 03 jun. 2018.

WORLD HEALTH ORGANIZATION (WHO). International Classification of diseases: revision 4. 1929. Disponível em: http://www.wolfbane.com/icd/icd4h.htm. Acesso em: 03 jun. 2018.

WORLD HEALTH ORGANIZATION (WHO).

International Classification of diseases: revision 5. 1938. Disponível em: http://www.wolfbane.com/icd/icd5h.htm. Acesso em: 03 jun. 2018.

WORLD HEALTH ORGANIZATION (WHO).

International Classification of diseases: revision 6. 1948a. Disponível em: http://www.wolfbane.com/icd/icd6.htm. Acesso em: 03 jun. 2018.

\section{WORLD HEALTH ORGANIZATION (WHO).}

International Classification of diseases: revision 6. $1948 \mathrm{~b}$. Disponível em: http://www.wolfbane.com/icd/icd6h.htm. Acesso em: 03 jun. 2018.

WORLD HEALTH ORGANIZATION (WHO). International Classification of diseases: revision 7. 1955a. Disponível em: http://www.wolfbane.com/icd/icd7.htm. Acesso em: 03 jun. 2018.

WORLD HEALTH ORGANIZATION (WHO). International Classification of diseases: revision 7. 1955b. Disponível em: http://www.wolfbane.com/icd/icd7h.htm. Acesso em: 03 jun. 2018.

WORLD HEALTH ORGANIZATION (WHO). International Classification of diseases: revision 8. 1965a. Disponível em: http://www.wolfbane.com/icd/icd8.htm. Acesso em: 03 jun. 2018.

WORLD HEALTH ORGANIZATION (WHO). 
International Classification of diseases: revision 8. 1965b. Disponível em: http://www.wolfbane.com/icd/icd8h.htm. Acesso em: 03 jun. 2018.

\section{WORLD HEALTH ORGANIZATION (WHO).}

International Classification of diseases: revision 9. 1975a. Disponível em: http://www.wolfbane.com/icd/icd9.htm. Acesso em: 03 jun. 2018.

WORLD HEALTH ORGANIZATION (WHO).

International Classification of diseases: revision 9. 1975b. Disponível em: http://www.wolfbane.com/icd/icd9h.htm. Acesso em: 03 jun. 2018.

\section{WORLD HEALTH ORGANIZATION (WHO).}

International Classification of diseases: revision 10. 1990a. Disponível em: http://www.wolfbane.com/icd/icd10.htm. Acesso em: 03 jun. 2018.

\section{WORLD HEALTH ORGANIZATION (WHO).}

International Classification of diseases: revision 10. 1990b. Disponível em: http://www.wolfbane.com/icd/icd10h.htm. Acesso em: 03 jun. 2018.

\section{WORLD HEALTH ORGANIZATION (WHO).}

International Classification of diseases: revision 1. 1900. Disponível em: http://www.wolfbane.com/icd/icd1h.htm. Acesso em: 03 jun. 2018.

\section{WORLD HEALTH ORGANIZATION (WHO).}

International Classification of diseases: revision 11. 2018. Disponível em: https://icd.who.int/. Acesso em: 02 ago. 2018.

WORLD HEALTH ORGANIZATION (WHO). History of the development of the ICD. Disponível em: http://www. who.int/classifications/icd/en/HistoryOfICD.pdf. Acesso em: 03 ago. 2018. 